\title{
Coumarin derivative polymers for thermo- and light-responsive monofunctional polymers: Effect of additives on
} thermo-responsivity

\section{Eriko Sato}

\begin{tabular}{|c|l|}
\hline Citation & AIP Conference Proceedings, 2040(1); 020006 \\
\hline Issue Date & $2018-11-30$ \\
\hline Type & Conference paper \\
\hline Textversion & author \\
\hline \multirow{3}{*}{ Rights } & $\begin{array}{l}\text { C } 2018 \text { Author(s). Published by AIP Publishing. } \\
\text { This article may be downloaded for personal use only. Any other use requires prior } \\
\text { permission of the author and AIP Publishing. This article appeared in AIP } \\
\text { Conference Proceedings. 2040, 020006 (2018) and may be found at } \\
\text { https://doi.org/10.1063/1.5079048 }\end{array}$ \\
\hline DOI & $10.1063 / 1.5079048$ \\
\hline
\end{tabular}

\author{
Self-Archiving by Author(s) \\ Placed on: Osaka City University
}

SATO E. (2018). Coumarin derivative polymers for thermo- and light-responsive monofunctional polymers: Effect of additives on thermo-responsivity. AIP Conference Proceedings. 2040. 020006. https://doi.org/10.1063/1.5079048 


\title{
Coumarin Derivative Polymers for Thermo- and Light- Responsive Monofunctional Polymers: Effect of Additives on Thermo-Responsivity
}

\author{
Eriko Sato \\ Department of Applied Chemistry and Bioengineering, Graduate School of Engineering, \\ Osaka City University, 3-3-138 Sugimoto, Sumiyoshi, Osaka 558-8585, Japan \\ ${ }^{\text {a)} C o r r e s p o n d i n g ~ a u t h o r: ~ s a t o @ a-c h e m . e n g . o s a k a-c u . a c . j p ~}$
}

\begin{abstract}
Methacrylic and acrylic homopolymers containing a coumarin unit in the side-chain, which undergoes reversible [2+2] cycloaddition by photoirradiation, are the very limited examples of the dual-stimuli responsive monofunctional polymers. The coumarin derivative polymers show lower critical solution temperature-type phase separation in some chlorinated organic solvents such as chloroform. In this study, the control of the cloud points of the coumarin derivative polymers by the addition of low-molecular-weight additives to the solution was investigated. It was revealed that the addition of low-molecular-weight compounds, which would interact with the coumarin derivative polymers, such as coumarin, 3,4-dihydrocoumarin, chromone, and 9-bromoanthracene resulted in significant increase in the cloud points. It was also found that the cloud points of the coumarin derivative polymer can be controlled by the addition amount of the additives up to near the boiling point of the solvent.
\end{abstract}

\section{INTRODUCTION}

Multi-stimuli responsive polymers have attracted much attention not only as functional materials but also from scientific point of view [1-3]. Most of multi-stimuli responsive polymers contain multi-functional groups which correspond to individual stimuli, and there are only a limited number of reports for the multi-stimuli responsive polymers containing a single functional group which correspond to more than two stimuli $[4,5]$. We discovered that the homopolymers of (meth)acrylates having a coumarin derivative unit in the side-chain ester group exhibited lower critical solution temperature (LCST)-type phase separation in several chlorinated organic solvents (Figure 1(a)) [6]. Coumarin derivatives also undergo reversible [2+2] cycloaddition (Fig. 1(b)), and thus we named the

(a)

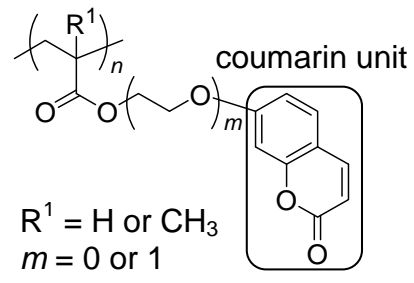

(b)<smiles>Cc1ccc2ccc(=O)oc2c1</smiles>

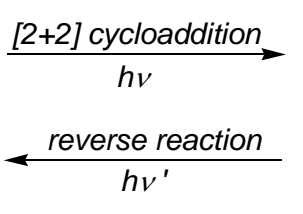<smiles>Cc1ccc2c(c1)OC(=O)C1C(=O)Oc3cc(I)ccc3C21</smiles>

FIGURE 1. (a) Structure of the poly(meth)acrylates containing a coumarin derivative unit showing LCST and (b) [2+2] cycloaddition and the reverse reaction of coumarin derivatives. 
coumarin derivative polymers "monofunctional dual-stimuli-responsive polymers", where the single functional group, i.e., the coumarin derivative unit, has the functions of both thermo- and photo-responsivities. The thermoresponsive behavior of the coumarin derivative polymers can be simply controlled by photoirradiation [6]. The thermoresponsive properties were also tuned by the copolymerization of the coumarin derivative monomers with solvophilic or solvophobic monomers [7].

It has been reported that third components, which interact with polymer chains, affect the thermoresponsive behavior of the polymer solution. The sets of the ternary-amine polymer with the trivalent counterion [8], the urea polymer with hydrogen bondable low-molecular-weight compounds [9], and the donner polymer with the accepter molecule [10] have been reported. These interactions between polymers and additives are relatively strong physical interactions. LCST-type phase separation of the coumarin derivative polymers are explained by the disappearance of the $\mathrm{CH}-\pi$ interaction between polymer side-chain and a polarized $\mathrm{C}-\mathrm{H}$ bond in chloroform [6]. In this study, the effects of the low-molecular-weight-additives, which would interact with polymer side-chains by relatively weak interactions such as $\pi-\pi$ and $\mathrm{CH}-\pi$ interactions, on the thermoresponsive behavior of coumarin derivative polymers were investigated.

\section{EFFECTS OF ADDITIVES}

Poly(7-(2-methacryloyloxyethoxy)coumarin) (PMEC) $\left(\mathrm{R}^{1}=\mathrm{CH}_{3}, m=1\right.$ in Fig. 1(a)) is one of the coumarin derivative polymers showing LCST-type phase separation in some chlorinated organic solvents including chloroform and deuterated chloroform. The addition effects of low-molecular-weight compounds, which would interact with the polymer side-chain by $\pi-\pi$ and/or $\mathrm{CH}-\pi$ interactions, to the solution of the coumarin derivative polymers, were investigated. A value of $T_{\mathrm{c}}$ is one of useful indexes to systematically compare LCST-type phase separation behavior. In this paper, the $T_{\mathrm{c}}$ values of polymer solutions were determined by the transmittance measurement, which is one of the well-established methods to experimentally evaluate $T_{\mathrm{c}}$ values [11]. Coil-toglobule transition of polymers showing LCST-type phase separation in solution can be monitored by optical transmittance at certain wavelength at elevated temperature. The $1.0 \mathrm{wt} \%$ of PMEC deuterated chloroform solutions containing the 3 equivalents of each additive relative to the MEC unit in PMEC were subjected to the transmittance measurements at the constant heating rate, $0.2^{\circ} \mathrm{C} \mathrm{min}^{-1}$. Transmittance (at $500 \mathrm{~nm}$ )-temperature plots for the PMEC solutions without additives and with coumarin as an additive are shown in Fig. 2 as the representative data. The transmittance of the solution at $500 \mathrm{~nm}$ is close to $100 \%$ in a coil state and steeply decreased to nearly $0 \%$ due to coil-to-globule transition. In this study, the temperature producing a $50 \%$ transmittance was defined as the cloud point $\left(T_{\mathrm{c}}\right)$. The $T_{\mathrm{c}}$ values in the presence of additives are summarized in Table 1 together with that of PMEC without additives. The difference in the $T_{\mathrm{c}}$ values $\left(\Delta T_{\mathrm{c}}\right)$ was defined as follows, $\Delta T_{\mathrm{c}}=T_{\mathrm{c}}$ (with additive) $-T_{\mathrm{c}}$ (without additive). As shown in Fig. 2 and Table 1, the addition of coumarin to PMCE solution resulted in a significant increase in $T_{\mathrm{c}}$, where $\Delta T_{\mathrm{c}}$ was $15^{\circ} \mathrm{C}$. The addition of 3,4-dihydrocoumarin and chromone having similar skeleton structures to coumarin were also resulted in significant increases in $T_{\mathrm{c}}$ values. In the case of 9-bromoanthracene and

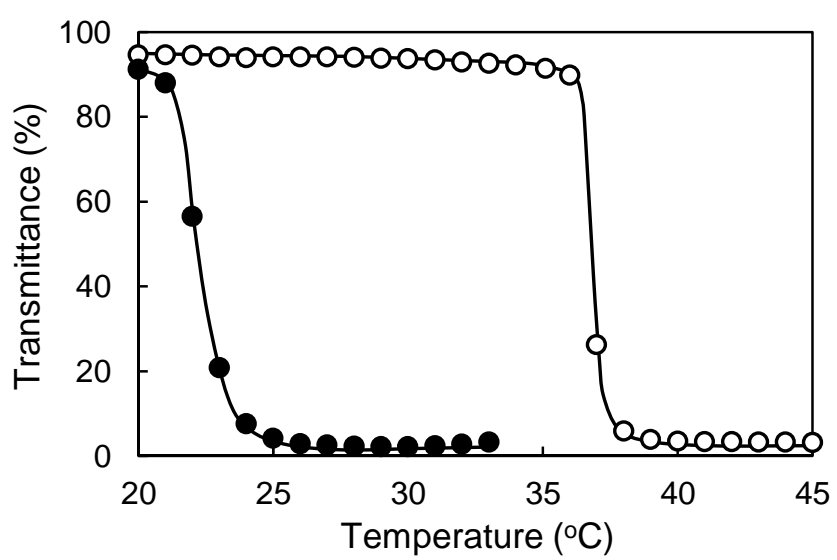

FIGURE 2. Temperature dependence of transmittance at $500 \mathrm{~nm}$ for the $1.0 \mathrm{wt} \%$ PMEC solutions ( $\bullet$ ) and the $1.0 \mathrm{wt} \%$ PMEC solutions containing 3 equivalent coumarin to MEC units in PMEC ( $O$ ) in deuterated chloroform. Solid curves were drawn as visual guides. 
1-bromonaphthalene, the $T_{\mathrm{c}}$ values were also increased, although the $\Delta T_{\mathrm{c}}$ value of 1-bromonaphthalene vas not very large. In order to consider the role of the additives, the solubilities of PMEC in some additives were examined. PMEC was soluble in melt coumarin, m.p. of which is $68-72{ }^{\circ} \mathrm{C}$, and was not soluble in 1-bromonaphthalene. These results show that the additives, which increased $T_{\mathrm{c}}$ of PMEC, did not simply act as good solvents.

Methyl cinnamate, which has the ring-opened structure of coumarin, and benzyl cinnamate as cinnamate derivatives were added to the PMEC solution, and marked decreases in the $T_{\mathrm{c}}$ values were obtained (Table 1). Phthalates, i.e., dimethyl phthalate, di-n-octyl phthalate, butyl benzyl phthalate, and dimethyl terephthalate, were also examined as compounds consisting of aromatic ring and polar ester groups, and all resulted in decreases in the $T_{\mathrm{c}}$ values except for dimethyl phthalate, where the $T_{\mathrm{c}}$ value did not change. In the case of dimethyl phthalate, in spite of the insolubility of PMEC in the additive, the $T_{\mathrm{c}}$ value did not change. The result also suggests that the roles of the additives are not just good or poor solvent. Furthermore, these results indicate that aromatic compounds having neighboring ring structures are essential to increase the $T_{\mathrm{c}}$ values of coumarin derivative polymers.

The addition effect of the low-molecular-weight additive, which increases the $T_{\mathrm{c}}$ value, to the coumarin derivative polymer solutions was further investigated in terms of the addition amount of the additive. The addition amount of 7-methacryloyloxycoumarin (MC) as the low-molecular-weight additive relative to the monomer units in poly(7-methacryloyloxycoumarin) (PMC) $\left(\mathrm{R}^{1}=\mathrm{CH}_{3}, m=0\right.$ in Fig. 1(a)) as the coumarin derivative polymer was changed from 0.1 to 100 equivalents in the $0.1 \mathrm{wt} \%$ PMC solution in the distilled chloroform. The $T_{\mathrm{c}}$ values tended to increase with increasing the addition amount of $\mathrm{MC}$, and in the presence of 100 equivalents of $\mathrm{MC}$, the solution was transparent even near the boiling point of chloroform $\left(=61^{\circ} \mathrm{C}\right)$. The result demonstrated that the $T_{\mathrm{c}}$ values of coumarin derivative polymers can be controlled from the original value, i.e., the $T_{\mathrm{c}}$ value in the absence of additives, to the boiling point of the solvent by simply adding the specific additives. The effects of the photoreaction of the coumarin unit on the thermos-responsivity are currently under investigation and will be reported elsewhere.

TABLE 1. Effect of additives on the $T_{\mathrm{c}}$ values. $^{a}$

\begin{tabular}{lcccc}
\hline Additives & m.p. $\left({ }^{\circ} \mathrm{C}\right)$ & $T_{\mathrm{c}}\left({ }^{\circ} \mathrm{C}\right)$ & $\Delta T_{\mathrm{c}}\left({ }^{\circ} \mathrm{C}\right)$ & $\begin{array}{c}\text { Solubility of } \\
\text { PMEC }^{b}\end{array}$ \\
\hline None & - & 22 & - & - \\
Coumarin & $68-72$ & 37 & 15 & soluble $^{c}$ \\
3,4-Dihydrocoumarin & 22 & 33 & 11 & n.d. \\
Chromone & $55-60$ & 32 & 10 & n.d. \\
9-Bromoanthracene & $97-100$ & 29 & 7 & n.d. \\
1-Bromonaphthalene & $2-4$ & 24 & 2 & insoluble \\
Methyl cinnamate & $36-38$ & 14 & -8 & n.d. \\
Benzyl cinnamate & 36 & 12 & -10 & n.d. \\
Dimethyl phthalate & -0.8 & 22 & 0 & insoluble \\
Di- $n$-octyl phthalate & -40 & $<10$ & $<-12$ & insoluble \\
Butyl benzyl phthalate & -40 & $<10$ & $<-12$ & insoluble \\
Dimethyl terephthalate & 142 & 13 & -11 & n.d. \\
\hline
\end{tabular}

${ }^{a}$ The 3 equivalents of additives relative to the MEC unit in PMEC were added to $1.0 \mathrm{wt} \%$ PMEC deuterated chloroform solution. ${ }^{b}$ Solubility of PMEC in an additive. ${ }^{c}$ Solubility of PMEC in melt coumarin.

\section{SUMMARY}

The addition effect of various aromatic low-molecular-weight compounds to the coumarin derivative polymers were investigated in terms of the LSCT-type phase separation behavior. Aromatic compounds having a neighboring ring structure such as coumarin, 3,4-dihydrocoumarin, chromone, 9-bromoanthracene, and 1-bromonaphthalene resulted in the increases in the $T_{\mathrm{c}}$ values of PMEC deuterated chloroform solution. The $T_{\mathrm{c}}$ values of the coumarin derivative polymer solution increased with increasing the addition amount of the coumarin derivative up to near the boiling point of the solvent. The control of $T_{\mathrm{c}}$ values of thermoresponsive polymers is important for their practical 
applications [12], thus the present results are expected to be useful to design functional materials based on the coumarin derivative polymers as stimuli-responsive polymers.

\section{REFERENCES}

1. P. Schattling, F. D. Jochum, and P. Theato, Polym. Chem. 5, 25-36 (2014).

2. S. Guragain, B. P. Bastakoti, V. Malgras, K. Nakashima, and Y. Yamauchi, Chem. - Eur. J. 21, 13164-13174 (2015).

3. J. Zhuang, M. R. Gordon, J. Ventura, L. Li, and S. Thayumanavan, Chem. Soc. Rev. 42, 7421-7435 (2013).

4. L. H. Gan, Y. Y. Gan, and G. R. Deen, Macromolecules 33, 7893-7897 (2000).

5. L. Ning, Y. Min, Z. Maolin, L. Jiuqiang, and H. Hongfei, Radiat. Phys. Chem. 61, 69-73 (2001).

6. E. Sato, Y. Masuda, J. Kadota, T. Nishiyama, and H. Horibe, Eur. Polym. J. 69, 605-615 (2015).

7. E. Sato, R. Nakanishi, T. Nishiyama, and H. Horibe, Chem. Lett. 46, 108-110 (2017).

8. F. A. Plamper, M. Ballauff, and A. H. E. Müller, J. Am. Chem. Soc. 129, 14538-14539 (2007).

9. S. Amemori, K. Kodado, and K. Sada, J. Am. Chem. Soc. 134, 8344-8347 (2012).

10. S. Amemori, K. Kokado, and K. Sada, Angew. Chem. Int. Ed. 52, 4174-4178 (2013).

11. A. Halperin, M. Kroger, and F. M. Winnik, Angew. Chem. Int. Ed. 54, 15342-15367 (2015).

12. I. Tan, F. Roohi, and M.-M. Titirici, Anal. Methods 4, 34-43 (2012). 